\title{
On Erlang Capacity of CDMA Systems
}

\author{
Samad S. Kolahi \\ Member, IEEE \\ Unitec Institute of Technology, Auckland, New Zealand
}

\begin{abstract}
In this paper, the Erlang capacity of mobile protocols such as FDMA (Frequency Division Multiple Access), TDMA (Time Division Multiple Access), and CDMA (Code Division Multiple Access) systems are compared assuming perfect power control. Methods of calculating the traffic capacity of such systems are discussed. A simulation and modeling method is used and the results are compared with previous analytical methods. The simulation result is close to analytical result although a different approach is adopted. CDMA can provide up-to 20 times more traffic capacity than FDMA and 5.3 times more traffic capacity than TDMA.
\end{abstract}

Key words: CDMA, Capacity, Erlang

\section{INTRODUCTION}

Mobile communications has enjoyed continuous growth in terms of number of mobile phone users in the last several years. Traffic management of the network is becoming more important as the number of mobile phones increase. Tele-traffic engineering issues are vital in planning, design, and dimensioning of mobile CDMA networks. Traffic engineering of FDMA and TDMA systems is a straightforward matter as each user occupies a slice of the bandwidth or time slot respectively. If each user occupies $30 \mathrm{KHz}$ of bandwidth, with frequency reuse of 7 and 3 sectors per cell, the number of channels per sector in $12.5 \mathrm{MHz}$ is 19 . For a TDMA system, the system uses the $30 \mathrm{KHz}$ band for three calls, each taking turns in using the $30 \mathrm{KHz}$ range. The number of channels will be $N=57$ Channels/sector. Using the ErlangB formula (1), assuming blocking probability of 0.02 , the traffic intensity is calculated as $A=12.3$ for FDMA systems and $A=46.8$ for TDMA systems. Note that the result implies that although the number of channels is 3 times greater in TDMA compared to FDMA, it can carry 3.8 times more traffic to that of FDMA.

The original version of this chapter was revised: The copyright line was incorrect. This has been corrected. The Erratum to this chapter is available at DOI: 10.1007/978-0-387-35618-1_37 


$$
P_{\text {blocking }}=\frac{(A)^{N} / N !}{\sum_{k=0}^{N}(A)^{k} / k !}
$$

The traffic engineering issue is not straightforward in CDMA as all calls use the same bandwidth (the whole bandwidth spectrum). The traffic capacity of the CDMA system can be investigated by varying the traffic loads (arrivals versus departures to the system) and determining the probability of call losses.

The organization of this paper is as follows. In the next section the system model is discussed. The blocking probability calculations are introduced in section 3. Simulation and analytical results are reported in section 4 and some concluding remarks are given in section 5 .

\section{SYSTEM MODEL}

A cellular CDMA network with 37 cells is considered (a home cell and three tiers of neighboring cells) with a base station located at the center of each cell. All cells are assumed to be homogeneous in every respect. The reverse link (from mobile to cell site) is modeled as it is the limiting link due to its inferior performance compared to forward link [1]. The calls to the CDMA system are modeled as Poisson [2] with mean arrival rate of $\lambda$ calls/sector/second and mean call holding time of $1 / \mu$ seconds per call. In queuing terms, this is a $\mathrm{M} / \mathrm{M} / \infty$ system which is being used for CDMA systems modeling. Once the mobile call has been admitted, it stays in the system during its call holding time which is modeled as negative exponential with probability density function:

$$
f(t)=\mu e^{-\mu t}
$$

Traffic density (offered traffic load), $\lambda / \mu$, represents the excess of the arrival rate versus departure rate. $\lambda / \mu$ is measured in Erlang.

All calls are allowed into the system (soft capacity) if they meet the required Quality of Service (QoS). Any calls not meeting this required quality are not permitted to enter the system but are blocked.

\section{CDMA BLOCKING PROBABILITY}

In CDMA all calls use the same frequency range. These calls therefore interfere with one another. CDMA capacity decreases with the amount of interference. Consider a CDMA home cell and its neighboring cells, each cell site not only receives interference signal from mobiles in the home cell 
(intra-cell interference) but also from mobiles located in neighboring cells (inter cell interference).

The power of signals received is the product of the transmitted power, $m$ th power of the distance and a lognormal shadowing parameter $(\xi)$ with mean zero and standard deviation of $\sigma_{\xi}$. This shadowing parameter varies with different terrains. Assuming $S_{\mathrm{t}}$ and $S$ are the transmitted and received power respectively, we have:

$$
S=S_{t} \cdot r^{-m} 10^{\xi / 10}
$$

The interference from the $j$ th mobile in neighboring cell $i$ is expressed as [4]:

$$
\begin{aligned}
& (I)_{i j}=S \frac{r_{m}^{m}}{10^{\xi_{m} / 10}} \cdot \frac{10^{\xi_{0} / 10}}{r_{0}^{m}} \\
& (I / S)_{i j}=\left(r_{m} / r_{0}\right)^{m} 10^{\xi_{0}-\xi_{m} / 10}
\end{aligned}
$$

where $S$ is the received signal strength at home base station, $r_{m}$ is the distance to corresponding home cell base station (figure 1), $r_{0}$ is the distance to the neighboring cell, $\xi_{0}$ and $\xi_{m}$ are lognormal (Gaussian in $\mathrm{dB}$ ) random variable distribution with zero mean and standard deviation $\sigma_{\xi}$ representing shadowing parameter in neighboring and home cell, and $m$ is path loss exponent.

Total other cell interference $I_{o}$ is interference produced by all users who are power controlled by other base stations. Assuming a CDMA system with $M$ outer cells and $N$ users per cell, then the total other user interferences-to-signal ratio $(I / S)_{o}$ is:

$$
(I / S)_{o}=\sum_{i=1}^{M} \sum_{j=1}^{N} I_{i j} / S
$$

On each arrival of a new call, the total interference is determined from which the blockage condition can be checked. This involves repeatedly generating $r_{m}$ between 0 and 1 and uniform random variable $\theta$ between 0 and $2 \pi$. Using figure $1, r_{0}$ can be calculated for each user as:

$$
r_{0}=\sqrt{r_{m}^{2}+d^{2}+2 d r_{m} \operatorname{Cos} \theta}
$$




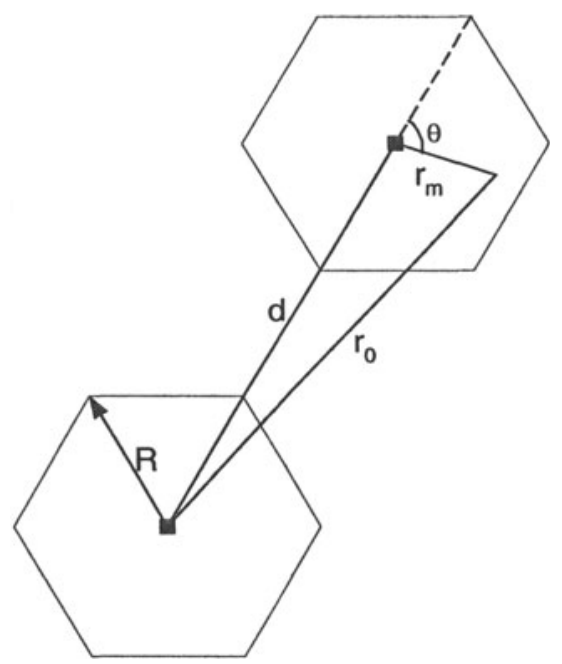

Figure 1. Interfering call distance to home cell

Using equations (5) and (6), the total received power from interfering cells at the home base station is calculated by considering the path loss exponent $m$ and shadowing parameter $\xi_{0}-\xi_{m}$. If independent lognormal variables $\xi_{m}$ and $\xi_{0}$ have average zero and variance of $\sigma_{\xi}{ }^{2}, \xi_{0}-\xi_{m}$ has mean zero and variance $2 \sigma_{\xi}{ }^{2}$. For each interfering call, a lognormal shadowing parameter, $\xi_{0}-\xi_{m}$, is generated with mean zero and standard deviation $\sigma_{\xi} \sqrt{2}$. The transmission quality of a CDMA call may then be calculated in terms of the energy per bit over total interference [4] spectral density $E_{b} / N_{o}$.

$$
\frac{E_{b}}{N_{0}}=\frac{S / R}{I / W}=\frac{S / R}{\left((N-1) S+I_{o}+\eta\right) / W}=\frac{W / R}{(N-1)+(I / S)_{o}+\eta / S}
$$

$(I / S)_{o}$ is the ratio of other cells interference to the received signal strength $(S)$ at home base station, $N$ is the number of active users in the cell, $\eta$ is background noise, $W / R$ is Processing Gain, $W$ is available spread bandwidth, and $R$ is data rate.

Taking voice activity into consideration, we have:

$$
\frac{E_{b}}{N_{0}}=\frac{W / R}{\alpha(N-1)+\alpha(I / S)_{o}+\eta / S}
$$


On each call arrival $E_{b} / N_{o}$ is determined $(6,9)$ to decide if the call is accepted to the system or is blocked. The blocking probability can then be calculated for a given traffic load.

\section{RESULTS}

\subsection{Simulation Results}

The simulation is performed for one million arrivals. On each arrival, the total interference at the home base station is determined and from this the blocking condition can be checked. If the required call quality of $E_{b} / N_{0} \geq 7 d B$, or $B E R<10^{-3}$, is not achieved, the call is blocked. The blocking probability is then calculated for a given Erlang traffic $(\lambda / \mu)$, by taking the ratio of the total number of blocking events to the total number of call arrivals.

For values of $\sigma_{\xi}=8 d B, \quad W / R=125, E_{b} / N_{0}=5.012(7 d B)$, $\alpha=0.4, S / \eta=-1 d B$, and $m=4$ and the blocking probability of $2 \%$, and assuming perfect power control, traffic intensity $(\lambda / \mu)$ is determined by simulation as 27 Erlangs which corresponds to approximately 36 voice channels per sector per $1.25 \mathrm{MHz}$. The $12.5 \mathrm{MHz}$ spectrum utilizes nine $1.25 \mathrm{MHz}$ CDMA frequency blocks [5]. The number for channels must therefore be multiplied by 9 . Total number of channels supported will be 324 which corresponds to 309.7 Erlangs/sector.

\subsection{Analytical Results}

Using the analytical method [3] with perfect power control, the traffic capacity can be calculated as:

$$
\frac{\lambda}{\mu}=\frac{(1-\tau)(W / R) F\left(B, \sigma_{p}\right)}{\alpha(1+f)\left(E_{b} / N_{0}\right)} \text { Erlangs/sector }
$$

where

$$
B=\frac{\left(E_{b} / N_{0}\right)\left[Q^{-1}\left(P_{\text {blocking }}\right)\right]^{2}}{(W / R)(1-\tau)}
$$

and

$$
Q(z)=\int_{z}^{\infty} \frac{1}{\sqrt{2 \pi}} e^{\frac{-y^{2}}{2}} d y
$$


for perfect power control:

$$
F(B, 0)=1+\frac{B}{2}\left(1-\sqrt{1+\frac{4}{B}}\right)
$$

where $\tau$ is the background (thermal) noise to total acceptable interference, $\sigma_{p}$ is the standard deviation of power fluctuations, $f$ is the ratio of other cell interference to own cell interference. Numerically for $P_{\text {blocking }}=0.02$, $W / R=1250, \tau=0.1, \sigma_{p}=0, f=0.55, E_{b} / N_{0}=5.01(7 d B)$ and $\alpha=0.4$, we obtain: $\quad Q^{-1}\left(P_{\text {blocking }}\right)=2.057, \quad B=0.019, \quad F(B, 0)=0.871, \quad \lambda / \mu=315.3$ Erlangs/sector.

This analytical Erlang capacity of 315.3 is slightly higher but close to the simulation results of 309.7 Erlangs/sector obtained above.

Imperfections in power control schemes can reduce this capacity by approximately $20 \%$ [3]. The simulated CDMA traffic capacity is therefore approximately 247.8 Erlangs. This is $247.8 / 12.3=20$ times more than an analogue system and 5.3 times (247.8/46.8) more than the TDMA Erlang capacity calculated above. These results are very close to the results determined analytically in [3] although a different approach is adopted.

\section{CONCLUSIONS}

The traffic capacity of a CDMA system is analyzed using simulation and modeling tools and compared to analytical methods. The analytical results are slightly higher but close to simulation results obtained in this work.

CDMA can provide up to 20 times more traffic capacity than its predecessor FDMA systems and up to 5.3 times more traffic capacity than TDMA systems for the same frequency range.

\section{REFERENCES}

[1] J.S. Evans and D. Everitt, "Analysis of Reverse Link Traffic Capacity for Cellular Mobile Communication Networks Employing Code Division Multiple Access," Australian Telecommunication Networks and Applications Conference, Melbourne, pp. 775-780, 1994.

[2] J.S. Evans and D. Everitt, "On the Teletraffic Capacity of CDMA Cellular Networks," IEEE Transaction on Vehicular Technology, vol 48(1), pp. 153-165, 1999.

[3] A.M. Viterbi and A. J. Viterbi, "Erlang Capacity of a Power Controlled CDMA System," IEEE Journal on Selected Areas in Communications, vol 11(6), pp. 892-899, 1993.

[4] K.S. Gilhousen, I.M. Jacobs, R. Padovani, A. J. Viterbi , et al, "On the Capacity of a Cellular CDMA System," IEEE Transaction on Vehicular Technology, vol 40(2), pp. 303-311, 1991.

[5] R. Padovni, "Reverse Link Performance of IS-95 Based Cellular Systems," IEEE Personal Communications, third quarter, pp. 28-34, 1994. 